\title{
Exciton states and optical absorption in quantum wires under laser radiation
}

\author{
C. González-Santander and F Domínguez-Adame \\ GISC. Departamento de Física de Materiales, Universidad Complutense, E-28040 \\ Madrid, Spain
}

\begin{abstract}
We analyze the excitons states in a quantum wire under intense laser radiation. Electrons and holes are confined by the parabolic potential of the quantum wire. An exactly solvable model is introduced for calculating the exciton binding energy, replacing the actual Coulomb interaction between the electron and the hole by a projective operator.
\end{abstract}

Keywords: Excitons, quantum wires, optical absorption, laser radiation PACS: 71.35.-y, 73.21.Hb, 85.35.Be

The nature of exciton states has a significant influence on the optical properties of semiconducting nanostructures, like quantum dots and quantum wires (QWs). Quantum confinement effects arise when the size of the nanostructure is of the order of the exciton radius in the bulk semiconductor, leading to an enhancement of the exciton binding energy and an increase of the oscillator strength. As a consequence, optical absorption lines in semiconducting nanostructures are blue-shifted and carry information about the confinement of carriers. Most important, changes in the electronic and optical properties of nanostructures may be properly controlled by an appropriate selection of the sample geometry and material parameters, opening new potential applications in optoelectronics. In order to understand their dependence on material and geometry parameters, exciton states and binding energy have been calculated in a large number of different semiconducting nanostructures. The effective-mass approximation is usually taken as starting point to construct the Hamiltonian for the interacting electron-hole pair. Nevertheless, since no analytical solutions are available in many geometries of interest within this framework, the electronic states and the binding energy are often 
obtained by means of variational or numerical techniques $[1,2,3,4,5]$.

Therefore, and in view of the lack of analytical solutions of the exciton problem in QWs, in this work we consider an interacting electron-hole pair within the effective-mass framework. Since the operation of optoelectronic devices relies on the interaction of carriers with electromagnetic radiation, we also take into account the effects of an intense laser field on the exciton states. We address the study by introducing a solvable model in which the Coulomb potential between the electron and the hole is replaced by a nonlocal separable potential (NLP) $[6,7]$. In the case of semiconducting nanostructures in the absence of a laser field, the NLP method yields an exactly solvable envelope-function equation from which exciton states can be readily obtained with little computational effort $[8,9]$. Our aim in this paper is to show that analytical solutions can be obtained even if the QW is subjected to an intense laser field. To this end we consider the laser effects on both Coulomb potential (replaced by a NLP) and QW confinement potential [10].

We consider an electron-hole pair in a QW, oriented along the $Z$ axis, subjected to a laser field of frequency $\Omega$, whose vector potential is given by $\boldsymbol{A}(t)=A_{0} \widehat{\boldsymbol{e}}_{x} \cos \Omega t$. Here $\widehat{\boldsymbol{e}}_{x}$ is the unit vector along the $X$ axis. Following Refs. [10, 11, 12], in the high frequency limit, the Hamiltonian of the interacting electron-hole pair is given by

$$
\mathcal{H}=\sum_{i=e, h}\left[\frac{\boldsymbol{p}_{i}^{2}}{2 m_{i}}+V_{i}^{d}\left(\boldsymbol{r}_{i}, \boldsymbol{\alpha}_{0}\right)\right]+V_{C}^{d}\left(\boldsymbol{r}_{e}-\boldsymbol{r}_{h}, \boldsymbol{\alpha}_{0}\right)
$$

with $\boldsymbol{\alpha}_{0}=\alpha_{0} \widehat{\boldsymbol{e}}_{x}$, where $\alpha_{0}=(8 \pi / \Omega c)^{2} \sqrt{I} e / m, m$ being the reduced effective mass and $I$ the laser intensity. The dressed parabolic confinement potential and Coulomb potential are defined as follows

$$
\begin{aligned}
V_{i}^{d}\left(\boldsymbol{r}_{i}, \boldsymbol{\alpha}_{0}\right) & =\frac{1}{4} m_{i} \omega^{2}\left[\left(\boldsymbol{r}_{i}-\boldsymbol{\alpha}_{0}\right)^{2}+\left(\boldsymbol{r}_{i}+\boldsymbol{\alpha}_{0}\right)^{2}\right] \\
V_{C}^{d}\left(\boldsymbol{r}_{e}-\boldsymbol{r}_{h}, \boldsymbol{\alpha}_{0}\right) & =-\frac{e^{2}}{2 \epsilon}\left[\frac{1}{\left|\boldsymbol{r}_{e}-\boldsymbol{r}_{h}-\boldsymbol{\alpha}_{0}\right|}+\frac{1}{\left|\boldsymbol{r}_{e}-\boldsymbol{r}_{h}+\boldsymbol{\alpha}_{0}\right|}\right] .
\end{aligned}
$$

It is convenient to separate the problem into center of mass and relative coordinates, defined by $\boldsymbol{r}=\boldsymbol{r}_{e}-\boldsymbol{r}_{h}$ and $\boldsymbol{R}=\left(m_{e} \boldsymbol{r}_{e}+m_{h} \boldsymbol{r}_{h}\right) / M$, where the total and reduced effectives masses are $M=m_{e}+m_{h}$ and $m=m_{e} m_{h} / M$, respectively. The total Hamiltonian (1) can be expressed as $\mathcal{H}=\mathcal{H}_{C M}+\mathcal{H}_{r}$ 
with

$$
\begin{aligned}
\mathcal{H}_{C M} & =\frac{P^{2}}{2 M}+\frac{1}{2} M \omega^{2}\left(X^{2}+Y^{2}+\alpha_{0}^{2}\right) \\
\mathcal{H}_{r} & =\frac{p^{2}}{2 m}+\frac{1}{2} m \omega^{2} \rho^{2}-\frac{e^{2}}{2 \epsilon}\left[\frac{1}{\left|\boldsymbol{r}+\boldsymbol{\alpha}_{0}\right|}+\frac{1}{\left|\boldsymbol{r}-\boldsymbol{\alpha}_{0}\right|}\right] .
\end{aligned}
$$

where $\boldsymbol{\rho}=(x, y)$, and $\boldsymbol{P}$ and $\boldsymbol{p}$ are the conjugate momenta of the coordinates $\boldsymbol{R}$ and $\boldsymbol{r}$. From Eq. (3b) it becomes apparent that the relative dynamics is equivalent to a single particle in a two-center Coulomb field. The separation between the two centers is $2 \alpha_{0}$, being proportional to $\sqrt{I}$.

Notice that the center-of-mass problem is exactly solvable since $\mathcal{H}_{C M}$ corresponds to a two-dimensional oscillator Hamiltonian. Thus, we will focus on the relative Hamiltonian $\mathcal{H}_{r}$ in what follows. The corresponding eigenfunctions of $\mathcal{H}_{r}$ cannot be expressed in terms of elementary functions. Aiming to introduce a solvable model, we replace the Coulomb potentials in (3b) by a sum of NLPs to obtain the envelope-function $|\chi\rangle$ from the effective-mass equation $[8,9]$

$$
\mathcal{H}_{r}|\chi\rangle \longrightarrow\left(\mathcal{H}_{0}+\mathcal{O}_{P}\right)|\chi\rangle=E|\chi\rangle
$$

with $\mathcal{H}_{0}=p^{2} / 2 m+m \omega^{2} \rho^{2} / 2$. The projective operator is defined as

$$
\mathcal{O}_{P} \equiv-\frac{g_{0} \hbar^{2}}{4 m}\left[\left|v_{+}\right\rangle\left\langle v_{+}|+| v_{-}\right\rangle\left\langle v_{-}\right|\right]
$$

$v_{ \pm}$will be referred to as shape functions and it will be specified later, and $g_{0}$ is named coupling constant. Equation (4) can be solved exactly for any arbitrary NLP, provided the eigenstates of the $\mathcal{H}_{0}$ are known. To compute the envelope-function $|\chi\rangle$, we consider the resolvent of the Hamiltonian $\mathcal{H}_{0}$ as follows

$$
|\chi\rangle=-\left(\mathcal{H}_{0}-E\right)^{-1} V_{\mathrm{NL}}|\chi\rangle=\frac{g_{0} \hbar^{2}}{4 m} \sum_{\mu} \sum_{s= \pm} \frac{|\mu\rangle\langle\mu|}{E_{\mu}-E}\left|v_{s}\right\rangle\left\langle v_{s} \mid \chi\right\rangle,
$$

where $|\mu\rangle$ denotes the eigenstates of $\mathcal{H}_{0}$ with eigenvalues $E_{\mu}$. Projecting onto the kets $\left|v_{ \pm}\right\rangle$and setting the determinant of the resulting matrix to vanish we arrive at

$$
\left[S_{+}(E)-1\right]\left[S_{-}(E)-1\right]=|C(E)|^{2}
$$


where for brevity we have introduced the following definitions

$$
\begin{aligned}
S_{ \pm}(E) & =\frac{g_{0} \hbar^{2}}{4 m} \sum_{\nu} \frac{1}{E_{\nu}-E}\left|\left\langle v_{ \pm} \mid \mu\right\rangle\right|^{2}, \\
C(E) & =\frac{g_{0} \hbar^{2}}{4 m} \sum_{\nu} \frac{1}{E_{\nu}-E}\left\langle v_{+} \mid \mu\right\rangle\left\langle\mu \mid v_{-}\right\rangle .
\end{aligned}
$$

This transcendental equation provides the exciton energy $E$ in the nanostructure in the presence of the laser field, for any arbitrary shape functions $v_{ \pm}$and coupling constant $g_{0}$.

Before we proceed further we show how the value of the coupling constant can be determined. To calculate $g_{0}$ we consider the free-exciton case, when there is no confinement due to the QW $(\omega \rightarrow 0)$ and the laser field is switched off $\left(\Omega \rightarrow 0\right.$ and $\left.\alpha_{0} \rightarrow 0\right)$. In this limiting case the exciton energy $E$ becomes $-\mathrm{Ry}^{*}$, where $\mathrm{Ry}^{*}=m e^{4} / 2 \epsilon^{2} \hbar^{2}$ is the effective exciton Rydberg in the bulk semiconductor. The resulting value of the coupling constant depends on the shape functions chosen. Hereafter we take Gaussian NLPs of the form

$$
v_{ \pm}(\boldsymbol{r})=\left(\frac{1}{\sqrt{\pi} a}\right)^{3} \exp \left(-\frac{\left(\boldsymbol{r} \pm \boldsymbol{\alpha}_{0}\right)^{2}}{a^{2}}\right),
$$

Following the procedure discussed in Ref. [9], the corresponding coupling constant is found to be

$$
\frac{2 \pi}{g_{0}}=\frac{\pi}{a}\left[\sqrt{\frac{\pi}{2}}-\frac{\pi}{2} \sqrt{\delta} \exp (\delta / 2) \operatorname{erfc}(\sqrt{\delta / 2})\right] .
$$

where $\operatorname{erfc}(z)$ is the complementary error function [13]. For brevity we have defined $\delta=\left(a / a^{*}\right)^{2}$, where $a^{*}$ is the effective Bohr radius of the exciton in three dimensions.

After having obtained the coupling constant, we now turn to the normalized eigenfunctions of $\mathcal{H}_{0}$, which are required to obtain the exciton energy in the QW when the laser field is switched on. The eingenfunctions can be factorized in cylindrical coordinates as follows

$$
\varphi_{n \ell k_{z}}(\boldsymbol{r})=R_{n \ell}(\rho) \frac{e^{i \ell \theta}}{\sqrt{2 \pi}} \frac{e^{i k_{z} z}}{\sqrt{2 \pi}},
$$

with quantum numbers $\ell=0, \pm 1, \pm 2 \ldots, n=0,1,2, \ldots$ and $k_{z}$. The axial function corresponding to a two-dimensional harmonic oscillator described 
by $\mathcal{H}_{0}$ is given by (see, e.g., Ref. [14])

$$
R_{n \ell}(\rho)=\sqrt{\frac{2 n !}{(n+|\ell|) !}} \frac{\rho^{|\ell|}}{\mathcal{L}^{|\ell|+1}} e^{-\rho^{2} / 2 \mathcal{L}^{2}} L_{n}^{|\ell|}\left(\rho^{2} / \mathcal{L}^{2}\right),
$$

where $\mathcal{L}=\sqrt{\hbar / m \omega}$ is the QW radius and $L_{n}^{|\ell|}$ denotes the generalized Laguerre polynomial [13]. The eigenenergies of the two-dimensional oscillator are $E_{n \ell}=\hbar \omega(2 n+|\ell|+1)$.

From Eq. (8) one finds that $S_{+}(E)=S_{-}(E) \equiv S(E)$ and $C(E)$ becomes real. Using (7) the ground state energy of the exciton is obtained by solving the equation $S(E)+C(E)=1$, which leads to

$$
\begin{aligned}
\frac{2 \pi}{g_{0}} & =\frac{1}{(1+\mu)^{2} \mathcal{L}} \exp \left(-\frac{2 \gamma^{2} \beta}{1+\mu}\right) \sum_{n \ell} \frac{2 n !\left[1+(-1)^{|\ell|}\right]}{(n+|\ell|) !}\left(\frac{\gamma \sqrt{2 \beta}}{1+\mu}\right)^{2|\ell|} \\
& \times\left(\frac{1-\mu}{1+\mu}\right)^{2 n} \frac{\exp \left(\mu \varepsilon_{n \ell}\right)}{\sqrt{\varepsilon_{n \ell}}} \operatorname{erfc}\left(\sqrt{\mu \varepsilon_{n \ell}}\right)\left[L_{n}^{|\ell|}\left(\frac{2 \gamma^{2} \beta}{1-\mu^{2}}\right)\right]^{2},
\end{aligned}
$$

where $2 \pi / g_{0}$ is given by (10). For brevity we have introduced the notation $\mu=a^{2} / 2 \mathcal{L}^{2}, \gamma=\alpha_{0} / a^{*}, \beta=\left(a^{*} / \sqrt{2} \mathcal{L}\right)^{2}$ and $\varepsilon_{n \ell}=2(2 n+|\ell|+1-E / \hbar \omega)$.

Figure 1 shows the exciton energy, in units of the effective Rydberg, as a function of the parameter $\gamma$, for several values of the QW radius, parameterized by $\beta$. We have taken $\delta=0.01$ but we have checked that the results remain almost unchanged when $\delta=0.005$. The inset shows that the exciton energy approaches the value in a bulk semiconductor $\left(E \rightarrow-R y^{*}\right)$ in wide QWs $\left(\mathcal{L} \gg a^{*}\right)$, as expected when the laser field is absent. The inset also shows that the energy increases when the radius of the QW decreases, indicating strong confinements effects. The effects of the intense laser field are similar, in the sense that the increase of the intensity is accompanied by a remarkable increase of the exciton energy. This effect is more pronounced for smaller QWs. Nevertheless, the exciton energy seems to reach a saturation value beyond a threshold laser intensity. The threshold intensity increases on decreasing the QW radius, as seen from Fig. 1.

Once the exciton energy has been calculated, we now focus on $\mathrm{HH}_{1} \rightarrow \mathrm{E}_{1}$ excitonic transitions due to the laser irradiation. To this end, we use the method introduced in Refs. [10, 15]. The linear absorption coefficient due to $\mathrm{HH}_{1} \rightarrow \mathrm{E}_{1}$ excitonic transitions can be cast in the form

$$
A\left(\hbar \omega_{P}\right) \propto \sum_{\eta}\left|\beta_{\eta}(\mathbf{0})\right|^{2} \delta\left(E_{\eta}-\hbar \omega_{P}\right)
$$




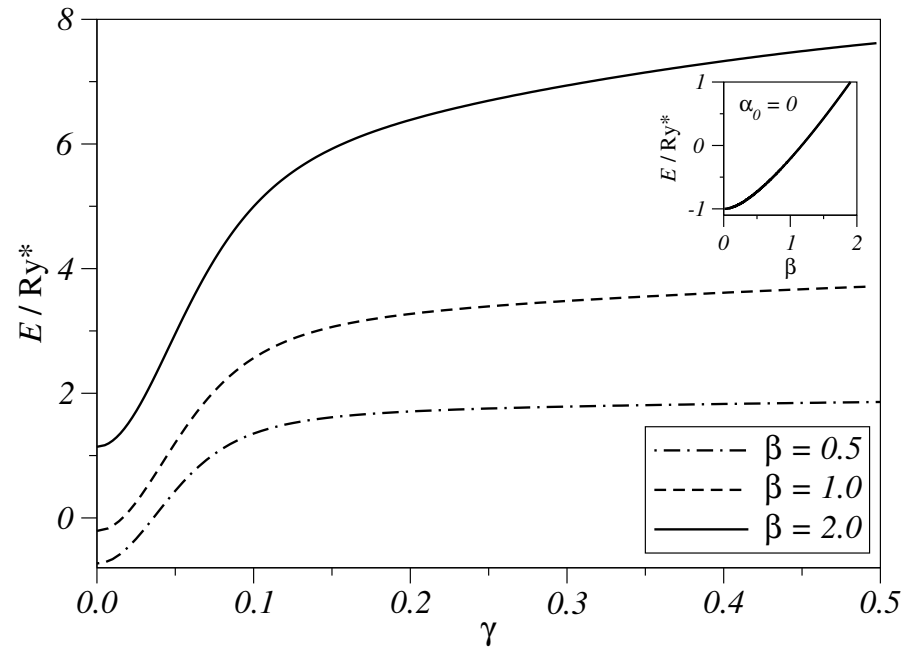

Figure 1: Exciton energy as a function of the parameter $\gamma=\alpha_{0} / a^{*}$. Several values of the QW radius are considered, the radius being parameterized by $\beta=\left(a^{*} / \sqrt{2} \mathcal{L}\right)^{2}$. The inset shows the exciton energy when the laser field is switched off.

where the index $\eta$ runs over the exciton states and $\beta_{\eta}(\boldsymbol{r})$ refers to the exciton envelope-function. Therefore, the term $\left|\beta_{\eta}(\mathbf{0})\right|^{2}$ gives the probability of finding the electron and the hole in the $\eta$ th excitonic state at zero distance from each other. Only excitons with non-zero amplitude at $\boldsymbol{r}=\mathbf{0}$ can absorb the light. The omitted prefactor gives rise to the standard selection rules within the dipolar approximation (the optical transitions are vertical in momentum space). Keeping only the lowest exciton state and replacing the $\delta$-function by a Lorentzian of width $\Gamma$ we obtain

$$
A\left(\hbar \omega_{P}\right) \propto \frac{1}{\left(E_{g}+2 \hbar \omega+M \omega^{2} \alpha_{0}^{2} / 2+E-\hbar \omega_{P}\right)^{2}+\Gamma^{2}},
$$

where we have used that $E_{\eta}=E_{h}^{e}+E$, with $E_{h}^{e}=E_{g}+2 \hbar \omega+M \omega^{2} \alpha_{0}^{2} / 2$ being the effective gap in the QW due to the dressed parabolic confinement potential and $E$ is the solution of Eq. (12). Figure 2 shows the normalized absorption coefficient in GaAs QWs with $\mathcal{L}=a^{*}$, as a function of the photon energy and $\gamma=\alpha_{0} / a^{*}$. The values of the physical parameters used in our calculations are $E_{g}=1.424 \mathrm{eV}, \Gamma=2 \mathrm{meV}, m_{e}=0.067 m_{0}, m_{h}=0.45 m_{0}$, where $m_{0}$ is the free electron mass. At low laser intensity (i.e. small $\gamma$ ) the absorption peak is strongly blue-shifted. Nevertheless, this shift is less pronounced at large $\gamma$, in agreement with the saturation effects discussed 
above (see Fig. 1).

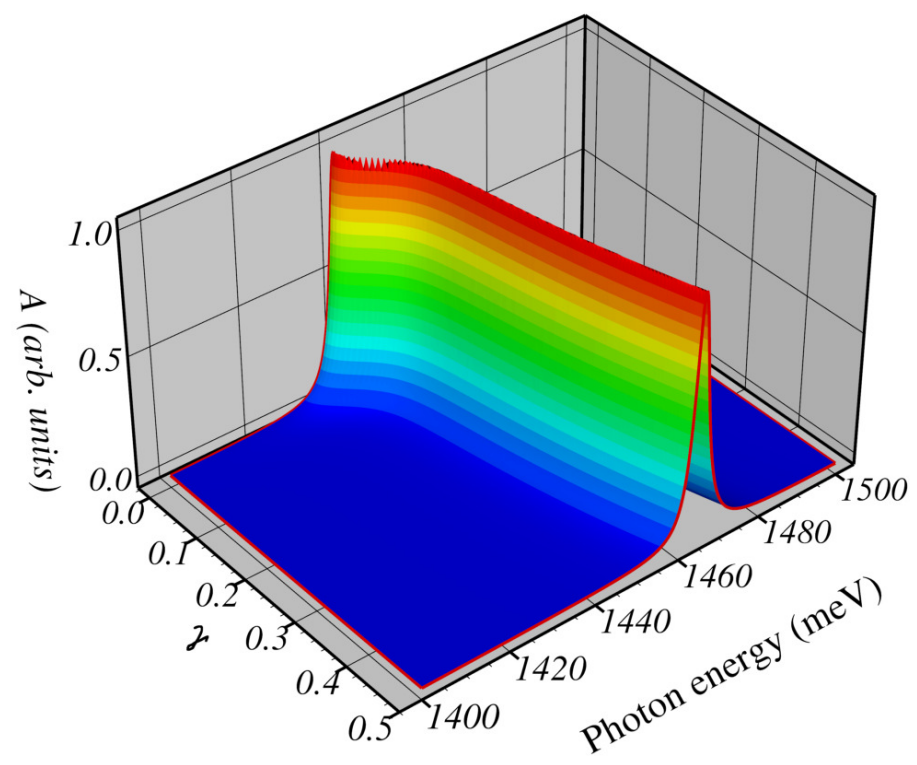

Figure 2: Normalized absorption coefficient in GaAs QWs as a function of the photon energy and $\gamma=\alpha_{0} / \mathcal{L}$.

In summary, we have presented a solvable model of excitons in QWs under laser irradiation. This model is based on the NLP approach, in which the dressed Coulomb interaction between the electron and the hole is replaced by a projective operator. This substitution is exact provided the appropriate shape functions $v_{ \pm}(\boldsymbol{r})$ are used. The exciton energy can be obtained for arbitrary shape functions. Once the solution is obtained, one can choose a potential shape function that reproduces the observed energy values. In this regard, we previously found that Gaussian shape functions provide accurate results in the absence of a laser field [9]. When the laser field is switched on, we found an increase of the exciton energy on increasing the laser intensity. The exciton energy reaches a saturation regime at very high laser intensity, the saturation value being larger for small QW radius. We also proved that this effect can be detected in the linear optical spectrum of the QW.

This work was supported by MEC (Project MOSAICO) and BSCH-UCM (Project PR34/07-15916). 
[1] J. Adrian Reyes, H. A. Coyotécatlb, M. Castillo-Mussotc and G. H. Cocoletzic, Physica E 15, 124 (2002).

[2] M. Combescot and T. Guillet, Eur. Phys. J. B 34, 9 (2003).

[3] E. W. S. Caetano, V. N. Freire, G. A. Farias and E. F. da Silva, Brazilian J. Phys. 34, 702 (2004).

[4] H. Hassanabadi, A. A. Rajabi, S. Zarrinkamar and M. M. Sarbazi, FewBody Syst. 45, 71 (2009).

[5] M. Santhi and A. J. Peter, Eur. Phys. J. B 71, 225 (2009).

[6] B. W. Knight and G. A. Peterson, Phys. Rev. 132, 1085 (1963).

[7] P. R. Sievert and M. L. Glasser, Phys. Rev. B 7, 1265 (1973).

[8] S. López and F. Domínguez-Adame, Semicond. Sci. Technol. 17, 227 (2002).

[9] C. Gonzalez-Santander and F. Domínguez-Adame, Physica E 41, 1645 (2009).

[10] H. Sari, E. Kasapoglu, I. Sökmen and M. Güneş, Phys. Lett. A 319, 211 (2003).

[11] H. Kramers, Collected Scientific Papers (North-Holland, Amsterdam, 1956), 866.

[12] M. Marinescu and M. Gavrila, Phys. Rev. A 53, 2513 (1995).

[13] M. Abramowitz and I. Stegun. Handbook of Mathematical Functions (Dover, New York, 1972).

[14] D. Chruściński, Ann. Phys. 321, 840 (2006).

[15] E. Kasapoglu, H. Sari, M. Bursal and I. Sökmen, Physica E 16, 237 (2003). 